\title{
Ensuring Effective Communication in the Diabetes Clinic: Creating a Curve of Remembering
}

\author{
Tejal Lathia ${ }^{1}$ \\ ${ }^{1}$ Department of Endocrinology, Fortis Hospital, Navi Mumbai, \\ Maharashtra, India
}

J Soc Health Diab 2019;7:22-24

\begin{abstract}
Address for correspondence Tejal Lathia, MD, DM, Department of Endocrinology, Fortis Hospital, Vashi 400703, Navi Mumbai, India (e-mail: drtblathia9748@gmail.com).
\end{abstract}
Abstract
Keywords
- information
- recall
- comprehension
- closed loop
- reinforcement

Patient-doctor interview involves exchange of information. Often, consultation may be hurried in resource-constrained settings whereby large quantity of health-related information is delivered to patient in a short period of time. Most people quickly forget what they have heard only once, thus putting into doubt how much of what patients are told, is really comprehended, recalled and thus, acted on. Repeating information, information delivery in staggered format, as well as the use of media for reinforcement helps patients comprehend and recall information better.

\section{Introduction}

Information imparted to a patient during the course of a consultation is voluminous. Diabetes mellitus (DM) is a multisystem disorder and greatly impacted by lifestyle. Thus, during the course of a consultation for DM, the doctor not only provides information about treatment options but also instructs the patient regarding diet and exercise. The doctor also discusses about foot care, need for annual screening investigations, instructions for follow-up, and so on

\section{Overworked Informant}

In resource-constrained settings, often the general practitioner or physician in a busy outpatient clinic is the sole person responsible for this task of imparting information. In some Southeast Asian countries, an average general practitioner or physician will see almost 30 to 40 patients a day. Thus, the busy practitioner is unlikely to repeat information. This is unlike many high-end centers who would have registered nurse practitioners, diabetic educators, and nutritionists where same information may be delivered by several people.

\section{The Natural History of Forgetting}

We usually remember information to which we turn our attention frequently and quickly forget information that we hear only once or twice. The Curve of Forgetting Graph or the Ebbinghuas curve ${ }^{1}$ developed in 1885 demonstrates how quickly we forget newly acquired information. When we talk to our patients, we often use words or terms they have never heard before.

Patients often hear the words HbA1c or glycosylated hemoglobin or diabetic retinopathy or microalbuminuria for the first time when they visit a specialist. How likely are the patients to remember these new terms is doubtful $(-$ Fig. 1).

According to the Curve of Forgetting, if we learn 10 new terms on a given day (very common situation in diabetic patients), we remember $100 \%$ at the time we first hear them. However, if we do not repeatedly hear these terms again, 24 hours later we forget $40 \%$ of these new terms. If we do not review these terms for another 24 hours, we forget $60 \%$ of the new terms.

Patients with diabetes and limited health literacy or numeracy are more likely to have poorer disease knowledge and symptom recognition, ${ }^{2,3}$ poorer glycemic control, ${ }^{4,5}$ greater difficulty interpreting food labels and estimating portion sizes, ${ }^{6,7}$ lower self confidence in diabetes management, fewer self-management behaviors, ${ }^{3,8}$ and poorer communication with their providers. ${ }^{9}$

Thus, poor recall due to the natural history of forgetting and poor comprehension due to limited health literacy, confound patient education greatly.

\section{Challenged Recipient}

Now, if we apply this same principle to a diabetic patient seeing a specialist for the first time, by the time the weekend received

March 28, 2019

accepted

April 6, 2019
DOI https://doi.org/

10.1055/s-0039-1692511

ISSN 2321-0656.
C2019 Novo Nordisk Education Foundation
License terms

(요 (1) $\odot \circledast$ 


\section{The curve of forgetting}

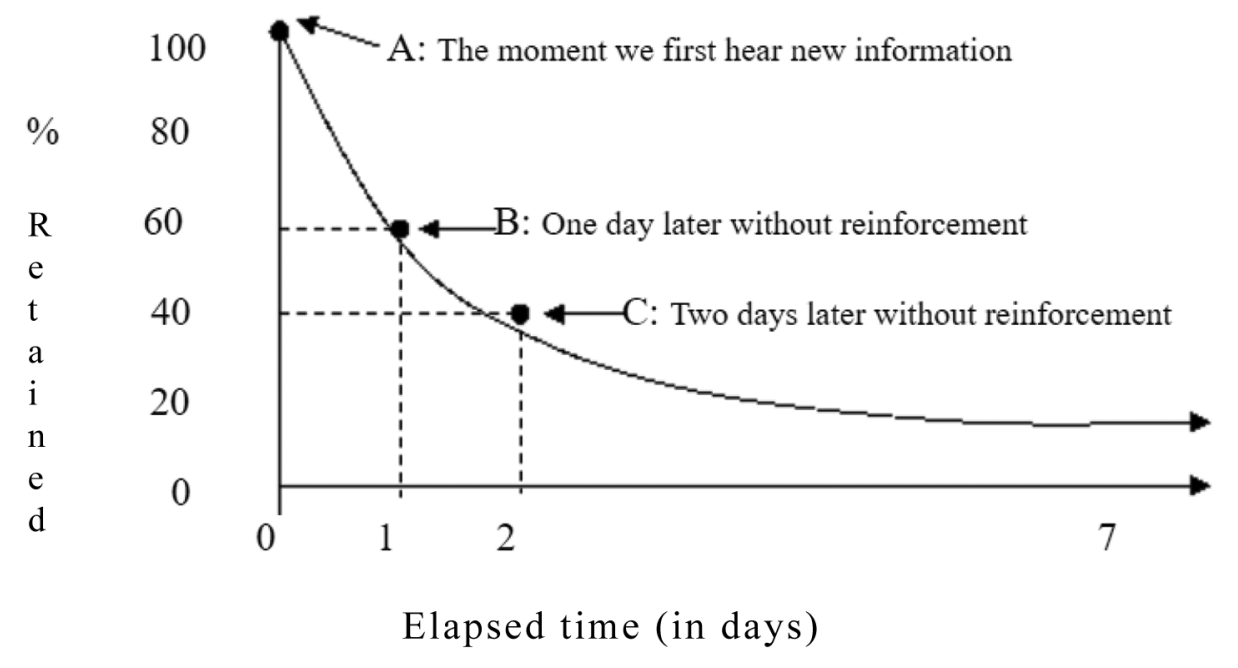

Fig. 1 The curve of forgetting.

is over, almost all of what information is imparted to the patient is lost. How can this situation be remedied?

\section{Improving Memory}

These are simple tools employable in day to day practice to ensure that patients can comprehend, recall, and act on information to achieve targets.

1. Short-term reinforcement or closing the loop.

2. Staggered information delivery.

3. Long-term reinforcement.

\section{Short-Term Reinforcement or Closing the Loop}

Short-term reinforcement is the most important for immediate effect. This means that at the end of the consultation, the doctor identifies three to four key messages that need to be remembered by the patient. The doctor repeats them to ensure that the patient has understood these messages and is able to carry out any instructions. After repeating the message, he assesses patient comprehension and recall. He clarifies any doubts and repeats the instructions.

For example, if after the consultation, the doctor wants to tell the patient that

1. He needs to exercise 30 minutes a day.

2. Be regular with his medications.

3. Complete his annual diabetic screening investigations before the next visit.

4. He needs the patient to bring HbA1c below $7 \%$.

Doctor T-So, Mrs V, we need to bring your HbA1c to below $7 \%$ from the current value of $7.6 \%$. For this I need you to commit to 30 minutes of walking or cycling daily for the next 3 months along with taking your medicines as instructed regularly. We also need you to do the listed investigations to ensure you have not developed any complications of diabetes. Is this clear?
Mrs.V-Yes Doctor.

Doctor T-Did you understand what I have said? Can you repeat for me what are the 4 things we need to accomplish before your next visit?

Mrs. V-Yes Doctor. Walk, take my meds, do my investigations before the next visit and ....I forgot the last one doctor.

Doctor T-And ensure an HbA1c below 7\% to prevent complications of diabetes. So, Mrs. V-Walk, take your meds daily, please complete all investigations before the next visit so that we can ensure your HbA1c is below 7\%.

Mrs. V-Ok, Doctor T. I will see you in 3 months with my reports and hopefully with an HbA1c below 7 .

Doctor T-If you walk daily and take your meds as you have promised Mrs. V, I am confident we will be able to achieve a target HbA1c below 7\%.

This is called closing the loop in business parlance. Closed loop communication is a communication technique used to avoid misunderstandings. When the sender gives a message, the receiver repeats this back. The sender then confirms the message by using the word "yes."

Schillinger et $\mathrm{al}^{10}$ conducted a study which observed the extent to which primary care physicians working in a public hospital assess patient recall and comprehension of new concepts during outpatient encounter. They used audiotapes of visits between 38 physicians and 74 English-speaking patients with DM and low functional health literacy. They examined whether there was an association between physician's application of interactive communication strategy and patients' glycemic control using information from clinical and administrative databases.

Physicians assessed recall and comprehension of any new concept in $20 \%$ of visits and for only $12 \%$ of new concepts. Patients whose physicians assessed recall or comprehension were more likely to have HbA1c below mean $(\leq 8.6 \%)$ versus patients whose physicians did not (odds ratio $[\mathrm{OR}]=8.96,95 \%$ confidence interval $[\mathrm{CI}]: 1.1-74.9 ; p=0.02$ ). After multivariate 
logistic regression, the two variables that independently associated with good glycemic control were higher health literacy levels ( $\mathrm{OR}=3.97,95 \% \mathrm{CI}: 1.09-14.47 ; p=0.04)$ and physicians' application of the interactive communication strategy $(\mathrm{OR}=15.15,95 \% \mathrm{CI}: 2.07-11.78 ; p<0.01)$.

\section{Staggered Information Delivery}

Delivering information in a staggered fashion is an effective way of imparting information. At each visit, one question is answered giving the patient time to digest the information, think about it, and come back next visit for a short review of previous sessions before new information is added. Thus, gradually building on information with a chance for doctor or educator to assess comprehension and recall. This helps to reinforce memory of the patient and retain the information.

1st visit-What is DM? Why does it happen?

2nd visit-Why do I need to control my blood sugars?

3rd visit-Medications for diabetes.

In a study on 170 subjects with type $2 \mathrm{DM}^{11}$ who were randomly assigned to group $(n=87)$ or individual $(n=83)$ educational setting, group information delivery was as good or slightly more effective at providing equivalent or slightly greater improvements in glycemic control. Thus, in resource-constrained settings, group information delivery based on a consistent, curriculum based but staggered way can be used to achieve better quality care.

\section{Long-Term Reinforcement}

In this age of social media and easy access to smart phones, long-term reinforcement is easy, provided the patient has access to accurate information from an expert instead of being diverted or confused with misinformation.

This can be done by follow-up telephone calls especially to new patients asking if instructions regarding medicine and lifestyle prescription are clear. This can usually be done in the week after the visit. Reminder telephonic messages with tips and tricks to manage DM help reinforce information. These can be disseminated in weekly or fortnightly fashion.

Lastly, applications that patients can download which have patient information videos and text can help the patient to clarify information at their leisure in a relaxed environment which is often not possible in the doctor's clinic. Reviewing information in a relaxed environment will also ensure better recall.

Diabetes self-management education (DSME) delivered via the internet is effective at improving measures of glycemic control and diabetes knowledge compared with usual care. ${ }^{12}$ Studies demonstrate that improved eating habits and increased attendance at clinic appointments occur after online DSME although engagement and usage of internet materials waned over time. Interactions that involve an element of interaction with healthcare providers were seen as attractive to participants.

\section{Conclusion}

Patient recall and comprehension are vital to ensure that patients achieve their glycemic goals. We forget $60 \%$ of new information by 48 hours. Thus, reinforcement of information is the key to information retention. Short-term reinforcement by "closing the loop" of the conversation, staggering of information with reassessment of recall at each step and using social media/smart phones for long-term reinforcement are simple tools we can employ to achieve treatment goals. This way, we can create a curve of remembering during the diabetes care dialogue.

\section{Conflict of Interest}

None declared.

\section{References}

1 Ebbinghaus H. Translation of Memory: A Contribution to Experimental Psychology. New York, NY: Dover Publications; 1987

2 Rothman RL, Malone R, Bryant B, et al. The spoken knowledge in low literacy in diabetes scale: a diabetes knowledge scale for vulnerable patients. Diabetes Educ 2005;31(2):215-224

3 Williams MV, Baker DW, Parker RM, Nurss JR. Relationship of functional health literacy to patients' knowledge of their chronic disease. A study of patients with hypertension and diabetes. Arch Intern Med 1998;158(2):166-172

4 Cavanaugh K, Huizinga MM, Wallston KA, Gebretsadik T, Shintani A, Davis D, Gregory RP, Fuchs L, Malone R, Cherrington A, Pignone M, DeWalt DA, Elasy TA, Rothman RL. Association of numeracy and diabetes control. Ann Intern Med 2008;148(10):737-746

5 Schillinger D, Grumbach K, Piette J, et al. Association of health literacy with diabetes outcomes. JAMA 2002;288(4):475-482

6 Huizinga MM, Carlisle AJ, Cavanaugh KL, et al. Literacy, numeracy, and portion-size estimation skills. Am J Prev Med 2009;36(4):324-328

7 Rothman RL, Housam R, Weiss H, et al. Patient understanding of food labels: the role of literacy and numeracy. Am J Prev Med 2006;31(5):391-398

8 Karter AJ, Subramanian U, Saha C, et al. Barriers to insulin initiation: the translating research into action for diabetes insulin starts project. Diabetes Care 2010;33(4):733-735

9 Arthur SA, Geiser HR, Arriola KR, Kripalani S. Health literacy and control in the medical encounter: a mixed-methods analysis. J Natl Med Assoc 2009;101(7):677-683

10 Schillinger D, Piette J, Grumbach K, et al. Closing the loop: physician communication with diabetic patients who have low health literacy. Arch Intern Med 2003;163(1):83-90

11 Rickheim PL, Weaver TW, Flader JL, Kendall DM. Assessment of group versus individual diabetes education: a randomized study. Diabetes Care 2002;25(2):269-274

12 Pereira K, Phillips B, Johnson C, Vorderstrasse A. Internet delivered diabetes self-management education: a review. Diabetes Technol Ther 2015;17(1):55-63Q1: Please confirm that given names (red), middle names (black) and surnames (green) have been identified correctly. 